\title{
Application of GRS method to evaluation of uncertainties of calculation parameters of perspective sodium-cooled fast reactor
}

\author{
A. Peregudov ${ }^{1}$, O. Andrianova ${ }^{1}$, K. Raskach ${ }^{1}$ and A. Tsibulya ${ }^{1}$ \\ ${ }^{1}$ Institute for Physics and Power Engineering, Bondarenko Square 1, Obninsk 244033, Kaluga \\ Region, Russia
}

\section{Introduction}

Evaluation of calculation errors due to uncertainties of neutron data and technological parameters (geometrical and material data) has become one of the important problems of reactor physics. The well-known approach to solve this problem is based on the use of sensitivity coefficients (e.g. Ref. [1]) of reactor calculation parameters of interest to the input data (neutron cross sections, geometrical and material data). The main advantage of this approach is that small computational times are necessary. On the other hand, there are certain drawbacks of the approach. Sensitivities are usually calculated by the first-order perturbation theory, so the linear approximation is applied. Furthermore, there should be used different types of the perturbation theory for different types of calculation parameters. The most commonly used type of the perturbation theory allows one to compute sensitivities of Keff. Other modifications of the theory are more difficult to implement and use in 3D calculations. It is particularly difficult to calculate sensitivities of spatially distributed calculation parameters like power density.

Recently, because of the very high computational capabilities of modern computers, another approach has attracted attention of reactor physicists (e.g. Ref. [2]). It is based on random sampling sets of input calculation data (neutron cross sections, geometrical and material data) and multiple recalculations of the reactor calculation parameters of interest. This gives sets of statistically distributed values for each calculation parameter. These sets can be then statistically processed to obtain mean values and variances of the calculation parameters.

This method is very easy to implement though multiple calculations imply that computational time could be considerable as compared with the sensitivity approach. On the other hand, in the GRS approach all types of calculation parameters are treated simultaneously and in the same manner: each run of a neutron transport code with randomly sampled input data yields random values of all the calculation parameters of interest, no matter how many such parameters are considered and which type each parameter belongs to. After a preset number of individual runs are performed calculation uncertainties of the parameters are simultaneously estimated. 
In this paper the GRS technique is applied to estimate uncertainties of calculation parameters, such as Keff, power density and stainless steel dose rate, of a perspective sodium-cooled fast reactor. These uncertainties are due to uncertainties of neutron cross sections and other input parameters of the reactor calculation model (geometrical and material data). Calculations were performed using the diffusion nodal code TRIGEX (Ref. [3]) and Monte Carlo code MMK (Ref. [4]). Group constants were calculated by CONSYST (Ref. [5]) on the base of the 299-group library ABBN (Ref. [6]). In the case of Keff, the results obtained with the GRS technique are compared with those obtained with the sensitivity approach.

\section{Sensitivity Approach Versus GRS Technique}

All calculation results have calculation uncertainties. There are three potential sources of the uncertainties: a) methodical biases, which accompany calculations with engineering codes; b) technological uncertainties associated with uncertainties of geometrical and material characteristics of reactor structural elements specified by their manufacturers; c) uncertainties of neutron constants. In this paper the calculation uncertainties of the last two types are considered.

\subsection{Sensitivity Approach}

Consider a calculation parameter $p$ (for example Keff) that is to be calculated using input data $\vec{\sigma}=\left\{\sigma_{i}\right\}$ with a covariance matrix $\hat{W}=\left\{w_{i, j}\right\}$. The sensitivity coefficient of the calculation parameter $p$ to the input parameter $\sigma_{i}$ is defined as

$$
S_{p, i}=\frac{\sigma_{i}}{p} \frac{\partial p}{\partial \sigma_{i}}
$$

Then, the uncertainty of $p$ due to the uncertainties of $\vec{\sigma}=\left\{\sigma_{i}\right\}$ can be obtained as follows:

$$
\delta_{p}^{2}=\sum_{i, j} s_{p, i} w_{i, j} s_{p, j}
$$

Theoretically, this technique can be applied to any reactor parameter. Yet, sensitivities of many integral parameters can be hardly found. To obtain sensitivities of spatially distributed parameters is even more difficult. Hence, this technique is mainly used in criticality calculations.

\subsection{GRS Technique}

This technique consists in using multiple calculations with randomly selected input parameters (neutron constants, geometrical and material data). In this approach there is no fixed vector of input data $\vec{\sigma}$ as in usual calculations. Instead, random vectors of input data are generated on the base of the covariance matrix $\hat{W}$ that complements these data. The total number $m$ of these vectors is defined so as to obtain statistically representative calculation results. Thus, this technique is particularly productive when applied along with engineering diffusion codes that work pretty fast. Yet, modern computers make it applicable for Monte Carlo calculations as well.

The main point of the technique consists in drawing random correlated values from the $n$ dimensional multivariate normal distribution

$$
f(\vec{\sigma})=\frac{1}{(2 \pi)^{n / 2} \operatorname{det}^{1 / 2}(\hat{W})} \exp \left[-\frac{1}{2}(\vec{\sigma}-\vec{\mu})^{t} \hat{W}^{-1}(\vec{\sigma}-\vec{\mu})\right],
$$


with the mean vector $\vec{\mu}$ and the covariance matrix $\hat{W}$. The covariance matrix is assumed to be symmetric and positive-definite.

The well-known procedure consists in finding matrix $\hat{L}$ such that

$$
\hat{W}=\hat{L} \hat{L}^{t}
$$

and applying the following equation

$$
\vec{\sigma}=\vec{\mu}+\hat{L} \vec{z}
$$

where $\vec{z}=\left\{z_{i}\right\}$ is the random vector with independent components $z_{i}$ randomly selected from the normal distribution with $E\left(z_{i}\right)=0$ and $D\left(z_{i}\right)=1$.

Eq. 4 is known as the Cholesky decomposition. The particular procedure of calculating elements of the matrix $\hat{L}$ can be easily found in literature.

Let $\vec{\sigma}_{j}, j=1, \ldots, m$ be the randomly selected vectors of input data. Then, multiple calculations with these vectors will produce random vectors of calculation parameters $\vec{p}_{m}=\left\{p_{k, m}\right\}$, which can be used for estimating their means, variances and covariances:

$$
\begin{gathered}
E\left(p_{k}\right)=<p_{k}>=\frac{1}{m} \sum_{j=1}^{m} p_{k, j} \\
D\left(p_{k}\right)=<p_{k}^{2}>-<p_{k}>^{2}=\frac{1}{m} \sum_{j=1}^{m} p_{k, j}^{2}-\left[\frac{1}{m} \sum_{j=1}^{m} p_{k, j}\right]^{2} \\
V\left(p_{k}, p_{l}\right)=<p_{k} p_{l}>-<p_{k}><p_{l}>=\frac{1}{m} \sum_{j=1}^{m} p_{k, j} p_{l, j}-\frac{1}{m} \sum_{j=1}^{m} p_{k, j} \times \frac{1}{m} \sum_{j=1}^{m} p_{l, j}
\end{gathered}
$$

The computational sequence of the GRS technique is shown in Fig. 1.

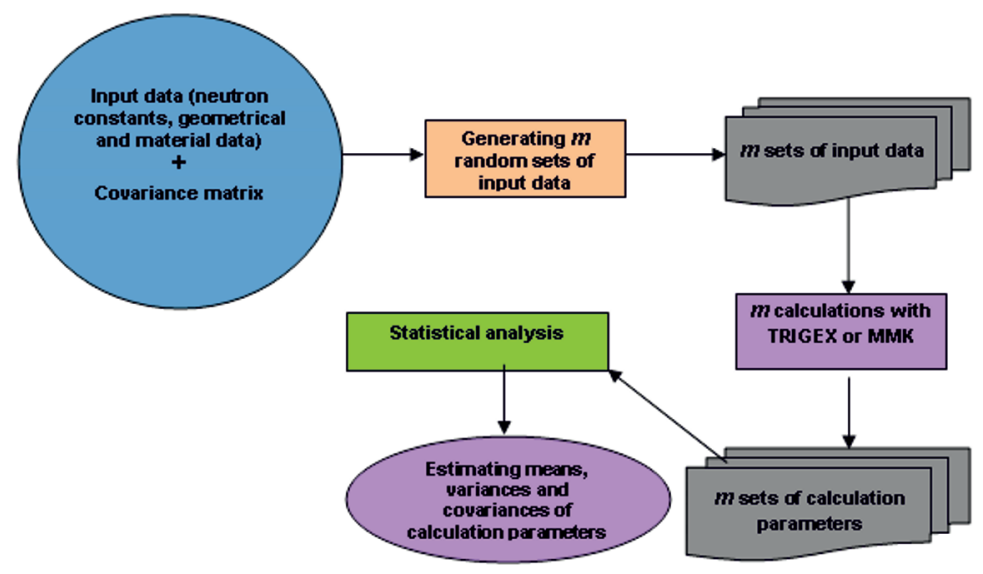

Fig. 1. Computational sequence of GRS technique. 


\section{Numerical results}

A description of the perspective sodium-cooled fast reactor investigated in this paper can be found in Ref. 7. So, only a brief description of this reactor is presented here. It has a core loaded with MOX fuel, axial and radial uranium blankets. Just above the core there is a sodium plenum. The core and the radial blanket (marked by dark blue color in Fig. 2) of the reactor are assembled from hexagonal (in plane) subassemblies. There are several groups of control rods, some of which are partly inserted into the core: emergency rods (marked by red color), reactivity compensation rods (marked by green color) and control rods (marked by blue color).

Calculations of the model were performed by the diffusion nodal code TRIGEX (Ref. [3]) and Monte Carlo code MMK (Ref. [4]) on the base of the 299-group library ABBN (Ref. [6]). Neutron constants were processed by CONSYST (Ref. [5]) that accounts for resonance self-shielding effect by the Bondarenko method.

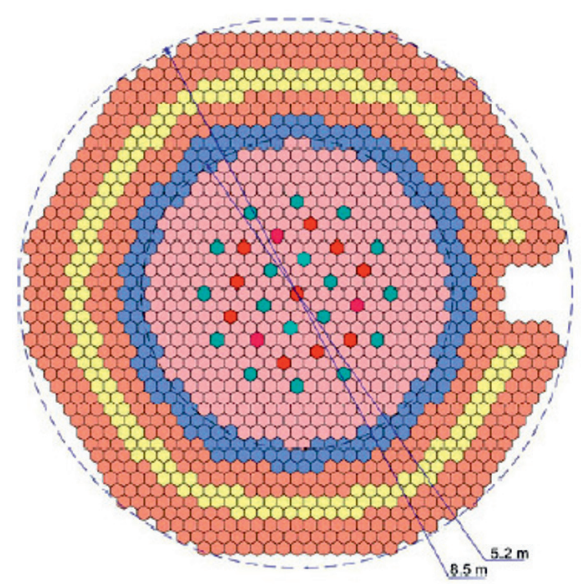

Fig. 2. Horizontal cross section of calculation.

The ABBN cross section library contains data on cross section covariances. These covariances were used to sample random sets of neutron cross sections. The cross sections for which uncertainties were taken into account are listed in Table I.

Table I. Neutron cross sections sampled to produce random sets of neutron data

\begin{tabular}{|l|l|l|}
\hline Nuclide / Element & Neutron cross section & Reaction number in ENDF notation \\
\hline${ }^{239} \mathrm{Pu}$ & $\sigma_{c}, \sigma_{f}, \bar{\nu}_{f}$ & $101,18,452$ \\
\hline${ }^{238} \mathrm{U}$ & $\sigma_{c}, \sigma_{i n}, \sigma_{e}$ & $101,4,2$ \\
\hline $\mathrm{Na}$ & $\sigma_{i n}, \sigma_{e}$ & 4,2 \\
\hline $\mathrm{Fe}$ & $\sigma_{c}, \sigma_{i n}$ & 101,4 \\
\hline
\end{tabular}

Table II gives technological parameters for which uncertainties were taken into account. This table also gives specific values of the uncertainties used in calculations.

Table III and Figs. 3-4 present the results of estimation of calculation uncertainty of Keff, power density and stainless steel dose rate due to uncertainties of the neutron cross sections, geometrical and material data mentioned in Tables I-II. 
Table II. Technological parameters sampled to produce random sets of geometrical and material data

\begin{tabular}{|l|l|}
\hline Parameter & Uncertainty (1 standard deviation), \% \\
\hline MOX fuel linear density & 0.5 \\
\hline Atomic fraction of ${ }^{239} \mathrm{Pu}$ in $\mathrm{Pu}$ & 1.0 \\
\hline Mass fraction of $\mathrm{PuO}_{2}$ in $\mathrm{MOX}$ fuel & 0.5 \\
\hline Atomic fraction of $\mathrm{Cr}$ in stainless steel & 5.0 \\
\hline Atomic fraction of $\mathrm{Ni}$ in stainless steel & 5.0 \\
\hline Atomic fraction of $\mathrm{Mn}$ in stainless steel & 20 \\
\hline Linear density of stainless steel & 0.5 \\
\hline Core height & 0.5 \\
\hline Subassembly pitch & 0.5 \\
\hline
\end{tabular}

Table III. Calculation uncertainties of Keff

\begin{tabular}{|l|l|l|l|l|l|l|l|}
\hline \multirow{2}{*}{$\begin{array}{l}\text { Source of } \\
\text { uncertainty }\end{array}$} & \multicolumn{3}{|l|}{ TRIGEX, GRS technique } & \multicolumn{2}{l|}{ TRIGEX, } & \multicolumn{3}{l|}{ MMK, GRS technique } \\
\cline { 7 - 9 } & $200{ }^{*}$ & 400 & 600 & sensitivity approach & 200 & 400 & 600 \\
\hline${ }^{239} \mathrm{Pu}$ & 1.31 & 1.33 & 1.32 & 1.23 & 1.33 & 1.34 & 1.33 \\
\hline${ }^{238} \mathrm{U}$ & 0.96 & 0.98 & 0.97 & 0.80 & 0.97 & 0.99 & 0.98 \\
\hline $\mathrm{Fe}$ & 0.31 & 0.29 & 0.30 & 0.18 & 0.33 & 0.31 & 0.31 \\
\hline $\mathrm{Na}$ & 0.08 & 0.08 & 0.09 & 0.09 & 0.08 & 0.09 & 0.09 \\
\hline${ }^{239} \mathrm{Pu},{ }^{238} \mathrm{U}, \mathrm{Fe}, \mathrm{Na}$ & 1.49 & 1.51 & 1.50 & 1.48 & 1.51 & 1.52 & 1.51 \\
\hline $\begin{array}{l}\mathrm{Geometrical} \text { and } \\
\text { material data }\end{array}$ & 0.61 & -- & -- & 0.59 & 0.60 & -- & - \\
\hline
\end{tabular}

${ }^{*}$ Number of sets of input data (neutron cross sections)

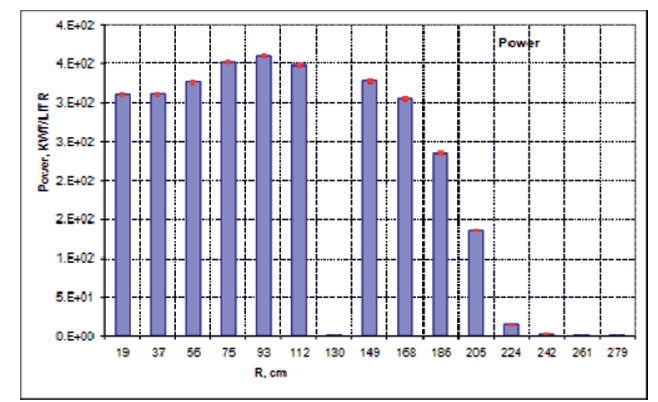

center of the core

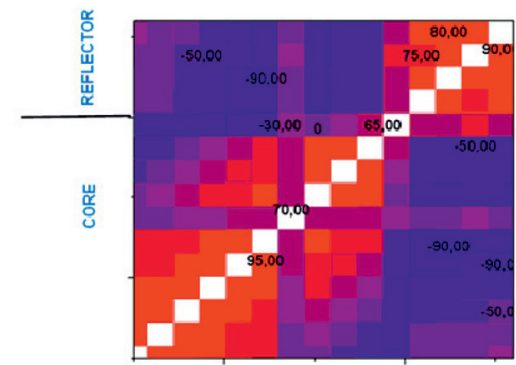

CORRELATION

Fig. 3.
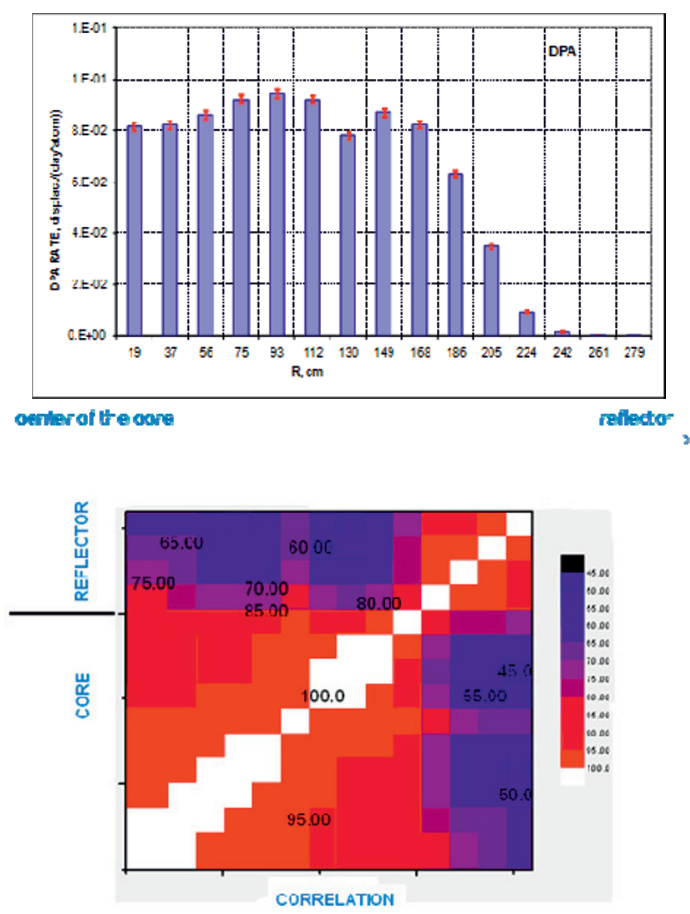

Fig. 4. 


\section{Conclusions}

In this work the GRS technique was used to estimate uncertainties of calculation parameters of a sodium-cooled fast reactor. The technique turned out to be easy to implement and quite robust at the same time. While there had been preliminary assumed that great numbers of sets of randomly sampled input data were necessary to reliably estimate uncertainties of output data, it was found that a few hundreds of such sets was usually sufficient (Table III). This conclusion is in agreement with the results obtained by earlier authors that considered other types of nuclear reactors. The method seems to be quite competitive with the sensitivity approach and in certain cases even preferable. The computational tool has been developed to automatically produce sets of randomly sampled input parameters, run neutron transport codes (TRIGEX or MMK) and perform statistical analysis of corresponding sets of output parameters.

\section{References}

1. B.T. Rearden, M.L. Williams et al. "Sensitivity and Uncertainty Analysis Capabilities and Data in SCALE", Nuclear Technology, Vol. 174, No. 2, 2011.

2. W. Zwermann et al. "Uncertainty Analyses with Nuclear Covariance Data in Reactor Core Calculations." Int. Conf. on Nuclear Data for Science and Technology 2010. Jeju Island, Korea, April 26-30, 2010.

3. A.S. Seregin, T.S. Kislitsina, A.M. Tsiboulia. TRIGEX.04 Code Package. Preprint IPPE-2846, Institute for Physics and Power Engineering, Obninsk (2000). (In Russian.)

4. A.A. Blyskavka, G.N. Manturov, M.N. Nikolaev, A.M. Tsiboulia. "Multigroup Monte Carlo Code MMK-KENO," Preprint IPPE-3145, Institute for Physics and Power Engineering, Obninsk (2009). (In Russian.)

5. "Multigroup Constant Set for Calculation of Neutron and Photon Radiation Fields and Functionals, Including the CONSYST2 Program," ORNL. RSICC DLC-182 (1995).

6. G.N. Manturov, M.N. Nikolaev, A.M. Tsiboulia, "ABBN-93 Group Data Library. Part 1: Nuclear Data for the Calculations of Neutron and Photon Radiation Fields." INDC (CCP)409/L, IAEA, p. 65 (1997).

7. V.M. Poplavsky et al. "Core Design and Fuel Cycle of Advanced Fast Reactor with Sodium Coolant." Int. Conf. on Fast Reactors and Related Fuel Cycles: Challenges and Opportunities (FR09), Kyoto, Japan, December 7-11, 2009. 